\title{
Possible Counterexample of the Riemann Hypothesis
}

\author{
Frank Vega
}

the date of receipt and acceptance should be inserted later

\begin{abstract}
Under the assumption that the Riemann hypothesis is true, von Koch deduced the improved asymptotic formula $\theta(x)=x+O\left(\sqrt{x} \times \log ^{2} x\right)$, where $\theta(x)$ is the Chebyshev function. On the contrary, we prove if there exists some real number $x \geq 10^{8}$ such that $\theta(x)>x+\frac{1}{\log \log \log x} \times \sqrt{x} \times \log ^{2} x$, then the Riemann hypothesis should be false. Note that, the von Koch asymptotic formula uses the Big $O$ notation, where $f(x)=O(g(x))$ means that there exists a positive real number $M$ and a real number $y$, such that $|f(x)| \leq M \times g(x)$ for all $x \geq y$. However, no matter how big we get the real number $y \geq 10^{8}$, the another positive real number $M$ could always prevail over the value of $\frac{1}{\log \log \log x}$ for sufficiently large numbers $x \geq y$.
\end{abstract}

Keywords Riemann hypothesis · Nicolas inequality $\cdot$ Chebyshev function · prime numbers

Mathematics Subject Classification (2010) MSC 11M26 · MSC 11A41 · MSC $11 \mathrm{~A} 25$

\section{Introduction}

The Riemann hypothesis is a conjecture that the Riemann zeta function has its zeros only at the negative even integers and complex numbers with real part $\frac{1}{2}$ [2]. The Riemann hypothesis belongs to the David Hilbert's list of 23 unsolved problems [2]. Besides, it is one of the Clay Mathematics Institute's Millennium Prize Problems [2]. This problem has remained unsolved for many years [2]. In mathematics, the Chebyshev function $\theta(x)$ is given by

$$
\theta(x)=\sum_{p \leq x} \log p
$$

F. Vega

CopSonic, 1471 Route de Saint-Nauphary 82000 Montauban, France

ORCiD: 0000-0001-8210-4126

E-mail: vega.frank@gmail.com 
where $p \leq x$ means all the prime numbers $p$ that are less than or equal to $x$. Say Nicolas $\left(p_{n}\right)$ holds provided

$$
\prod_{q \leq p_{n}} \frac{q}{q-1}>e^{\gamma} \times \log \theta\left(p_{n}\right) .
$$

The constant $\gamma \approx 0.57721$ is the Euler-Mascheroni constant, log is the natural logarithm, and $p_{n}$ is the $n^{\text {th }}$ prime number. The importance of this property is:

Theorem 1.1 [7], [8]. Nicolas $\left(p_{n}\right)$ holds for all prime numbers $p_{n}>2$ if and only if the Riemann hypothesis is true.

We know the following properties for the Chebyshev function:

Theorem 1.2 [11]. If the Riemann hypothesis holds, then

$$
\theta(x)=x+O\left(\sqrt{x} \times \log ^{2} x\right)
$$

for all $x \geq 10^{8}$

Theorem 1.3 [9]. For $2 \leq x \leq 10^{8}$

$$
\theta(x)<x
$$

We also know that

Theorem 1.4 [10]. If the Riemann hypothesis holds, then

$$
\left(\frac{e^{-\gamma}}{\log x} \times \prod_{q \leq x} \frac{q}{q-1}-1\right)<\frac{3 \times \log x+5}{8 \times \pi \times \sqrt{x}}
$$

for all numbers $x \geq 13.1$.

Let's define $H=\gamma-B$ such that $B \approx 0.2614972128$ is the Meissel-Mertens constant [6]. We know from the constant $H$, the following formula:

Theorem 1.5 [3].

$$
\sum_{q}\left(\log \left(\frac{q}{q-1}\right)-\frac{1}{q}\right)=\gamma-B=H .
$$

For $x \geq 2$, the function $u(x)$ is defined as follows

$$
u(x)=\sum_{q>x}\left(\log \left(\frac{q}{q-1}\right)-\frac{1}{q}\right) .
$$

We use the following theorems:

Theorem 1.6 [5]. For $x>-1$ :

$$
\frac{x}{x+1} \leq \log (1+x)
$$


Theorem 1.7 [4]. For $x \geq 1$ :

$$
\log \left(1+\frac{1}{x}\right)<\frac{1}{x+0.4}
$$

Let's define:

$$
\delta(x)=\left(\sum_{q \leq x} \frac{1}{q}-\log \log x-B\right) .
$$

Definition 1.8 We define another function:

$$
\varpi(x)=\left(\sum_{q \leq x} \frac{1}{q}-\log \log \theta(x)-B\right) .
$$

Putting all together yields the proof that the inequality $\varpi(x)>u(x)$ is satisfied for a number $x \geq 3$ if and only if $\operatorname{Nicolas}(p)$ holds, where $p$ is the greatest prime number such that $p \leq x$. In this way, we introduce another criterion for the Riemann hypothesis based on the Nicolas criterion and deduce some of its consequences.

\section{Results}

Theorem 2.1 The Riemann hypothesis is true if and only if the inequality $\varpi(x)>$ $u(x)$ is satisfied for all numbers $x \geq 3$.

Proof In the paper [8] is defined the function:

$$
f(x)=e^{\gamma} \times(\log \theta(x)) \times \prod_{q \leq x} \frac{q-1}{q} .
$$

We know that $f(x)$ is lesser than 1 when $\operatorname{Nicolas}(p)$ holds, where $p$ is the greatest prime number such that $2<p \leq x$. In the same paper, we found that

$$
\log f(x)=U(x)+u(x)
$$

where $U(x)=-\varpi(x)$ [8]. When $f(x)$ is lesser than 1 , then $\log f(x)<0$. Consequently, we obtain that

$$
-\varpi(x)+u(x)<0
$$

which is the same as $\varpi(x)>u(x)$. Therefore, this is a consequence of the theorem 1.1 .

Theorem 2.2 If the Riemann hypothesis holds, then

$$
\frac{3 \times \log x+5}{8 \times \pi \times \sqrt{x}+1.2 \times \log x+2}+\frac{\log x}{\log \theta(x)}>1
$$

for all numbers $x \geq 13.1$. 
Proof Under the assumption that the Riemann hypothesis is true, then we would have

$$
\prod_{q \leq x} \frac{q}{q-1}<e^{\gamma} \times \log x \times\left(1+\frac{3 \times \log x+5}{8 \times \pi \times \sqrt{x}}\right)
$$

after of distributing the terms based on the theorem 1.4 for all numbers $x \geq 13.1$. If we apply the logarithm to the both sides of the previous inequality, then we obtain that

$$
\sum_{q \leq x} \log \left(\frac{q}{q-1}\right)<\gamma+\log \log x+\log \left(1+\frac{3 \times \log x+5}{8 \times \pi \times \sqrt{x}}\right) .
$$

That would be equivalent to

$$
\sum_{q \leq x} \frac{1}{q}+\sum_{q \leq x}\left(\log \left(\frac{q}{q-1}\right)-\frac{1}{q}\right)<\gamma+\log \log x+\frac{3 \times \log x+5}{8 \times \pi \times \sqrt{x}+1.2 \times \log x+2}
$$

where we know that

$$
\begin{aligned}
\log \left(1+\frac{3 \times \log x+5}{8 \times \pi \times \sqrt{x}}\right) & <\frac{1}{\frac{8 \times \pi \times \sqrt{x}}{3 \times \log x+5}+0.4} \\
& =\frac{3 \times \log x+5}{8 \times \pi \times \sqrt{x}+0.4 \times(3 \times \log x+5)} \\
& =\frac{3 \times \log x+5}{8 \times \pi \times \sqrt{x}+1.2 \times \log x+2}
\end{aligned}
$$

according to theorem 1.7 since $\frac{8 \times \pi \times \sqrt{x}}{3 \times \log x+5} \geq 1$ for all numbers $x \geq 13.1$. We use the theorem 1.5 to show that

$$
\sum_{q \leq x}\left(\log \left(\frac{q}{q-1}\right)-\frac{1}{q}\right)=H-u(x)
$$

and $\gamma=H+B$. So,

$$
H-u(x)<H+B+\log \log x-\sum_{q \leq x} \frac{1}{q}+\frac{3 \times \log x+5}{8 \times \pi \times \sqrt{x}+1.2 \times \log x+2}
$$

which is the same as

$$
H-u(x)<H-\delta(x)+\frac{3 \times \log x+5}{8 \times \pi \times \sqrt{x}+1.2 \times \log x+2} .
$$

We eliminate the value of $H$ and thus,

$$
-u(x)<-\delta(x)+\frac{3 \times \log x+5}{8 \times \pi \times \sqrt{x}+1.2 \times \log x+2}
$$

which is equal to

$$
u(x)+\frac{3 \times \log x+5}{8 \times \pi \times \sqrt{x}+1.2 \times \log x+2}>\delta(x) .
$$


Under the assumption that the Riemann hypothesis is true, we know from the theorem 2.1 that $\varpi(x)>u(x)$ for all numbers $x \geq 13.1$ and therefore,

$$
\varpi(x)+\frac{3 \times \log x+5}{8 \times \pi \times \sqrt{x}+1.2 \times \log x+2}>\delta(x) .
$$

Hence,

$$
\frac{3 \times \log x+5}{8 \times \pi \times \sqrt{x}+1.2 \times \log x+2}>\log \log \theta(x)-\log \log x .
$$

Suppose that $\theta(x)=\varepsilon \times x$ for some constant $\varepsilon>1$. Then,

$$
\begin{aligned}
\log \log \theta(x)-\log \log x & =\log \log (\varepsilon \times x)-\log \log x \\
& =\log (\log x+\log \varepsilon)-\log \log x \\
& =\log \left(\log x \times\left(1+\frac{\log \varepsilon}{\log x}\right)\right)-\log \log x \\
& =\log \log x+\log \left(1+\frac{\log \varepsilon}{\log x}\right)-\log \log x \\
& =\log \left(1+\frac{\log \varepsilon}{\log x}\right)
\end{aligned}
$$

In addition, we know that

$$
\log \left(1+\frac{\log \varepsilon}{\log x}\right) \geq \frac{\log \varepsilon}{\log \theta(x)}
$$

using the theorem 1.6 since $\frac{\log \varepsilon}{\log x}>-1$ when $\varepsilon>1$. Certainly, we will have that

$$
\log \left(1+\frac{\log \varepsilon}{\log x}\right) \geq \frac{\frac{\log \varepsilon}{\log x}}{\frac{\log \varepsilon}{\log x}+1}=\frac{\log \varepsilon}{\log \varepsilon+\log x}=\frac{\log \varepsilon}{\log \theta(x)}
$$

Thus,

$$
\frac{3 \times \log x+5}{8 \times \pi \times \sqrt{x}+1.2 \times \log x+2}>\frac{\log \varepsilon}{\log \theta(x)} .
$$

If we add the following value of $\frac{\log x}{\log \theta(x)}$ to the both sides of the inequality, then

$$
\begin{aligned}
\frac{3 \times \log x+5}{8 \times \pi \times \sqrt{x}+1.2 \times \log x+2}+\frac{\log x}{\log \theta(x)} & >\frac{\log \varepsilon}{\log \theta(x)}+\frac{\log x}{\log \theta(x)} \\
& =\frac{\log \varepsilon+\log x}{\log \theta(x)} \\
& =\frac{\log \theta(x)}{\log \theta(x)} \\
& =1 .
\end{aligned}
$$

We know this inequality is satisfied when $0<\varepsilon \leq 1$ since we would obtain that $\frac{\log x}{\log \theta(x)} \geq 1$. Therefore, the proof is done. 
Theorem 2.3 If there exists some real number $x \geq 10^{8}$ such that

$$
\theta(x)>x+\frac{1}{\log \log \log x} \times \sqrt{x} \times \log ^{2} x,
$$

then the Riemann hypothesis is false.

Proof If the Riemann hypothesis holds, then

$$
\theta(x)=x+O\left(\sqrt{x} \times \log ^{2} x\right)
$$

for all $x \geq 10^{8}$ due to the theorem 1.2. Now, suppose there is a real number $x \geq 10^{8}$ such that $\theta(x)>x+\frac{1}{\log \log \log x} \times \sqrt{x} \times \log ^{2} x$. That would be equivalent to

$$
\log \theta(x)>\log \left(x+\frac{1}{\log \log \log x} \times \sqrt{x} \times \log ^{2} x\right)
$$

and so,

$$
\frac{1}{\log \theta(x)}<\frac{1}{\log \left(x+\frac{1}{\log \log \log x} \times \sqrt{x} \times \log ^{2} x\right)}
$$

for all numbers $x \geq 10^{8}$. Hence,

$$
\frac{\log x}{\log \theta(x)}<\frac{\log x}{\log \left(x+\frac{1}{\log \log \log x} \times \sqrt{x} \times \log ^{2} x\right)} .
$$

If the Riemann hypothesis holds, then

$$
\frac{3 \times \log x+5}{8 \times \pi \times \sqrt{x}+1.2 \times \log x+2}+\frac{\log x}{\log \left(x+\frac{1}{\log \log \log x} \times \sqrt{x} \times \log ^{2} x\right)}>1
$$

for those values of $x$ that complies with

$$
\theta(x)>x+\frac{1}{\log \log \log x} \times \sqrt{x} \times \log ^{2} x
$$

due to the theorem 2.2. By contraposition, if there exists some number $y \geq 10^{8}$ such that for all $x \geq y$ the inequality

$$
\frac{3 \times \log x+5}{8 \times \pi \times \sqrt{x}+1.2 \times \log x+2}+\frac{\log x}{\log \left(x+\frac{1}{\log \log \log x} \times \sqrt{x} \times \log ^{2} x\right)} \leq 1
$$

is satisfied, then the Riemann hypothesis should be false. Let's define the function

$$
v(x)=\frac{3 \times \log x+5}{8 \times \pi \times \sqrt{x}+1.2 \times \log x+2}+\frac{\log x}{\log \left(x+\frac{1}{\log \log \log x} \times \sqrt{x} \times \log ^{2} x\right)}-1 .
$$

The Riemann hypothesis is false when there exists some number $y \geq 10^{8}$ such that for all $x \geq y$ the inequality $v(x) \leq 0$ is always satisfied. We ignore when $2 \leq x \leq 10^{8}$ since $\theta(x)<x$ according to the theorem 1.3. We know that the function $v(x)$ is monotonically decreasing for every number $x \geq 10^{8}$. The derivative of $v(x)$ is negative for all $x \geq 10^{8}$. Indeed, a function $v(x)$ of a real variable $x$ is monotonically decreasing 
in some interval if the derivative of $v(x)$ is lesser than zero and the function $v(x)$ is continuous over that interval [1]. It is enough to find a value of $y \geq 10^{8}$ such that $v(y) \leq 0$ since for all $x \geq y$ we would have that $v(x) \leq v(y) \leq 0$, because of $v(x)$ is monotonically decreasing. We found the value $y=10^{8}$ complies with $v(y) \leq 0$. In this way, we obtain that $v(x) \leq 0$ for every number $x \geq 10^{8}$. Hence, the proof is complete.

\section{Appendix}

We found the derivative of $v(x)$ in the web site https: //www . wolframalpha.com/ input. Besides, we determine the sign of the function $v(x)$ using the tool $g p$ from the web site https://pari.math.u-bordeaux.fr. In the project PARI/GP, the method $\operatorname{sign}(F(X))$ returns -1 when the function $F(X)$ is negative in the value of $X$. We checked that is negative for $X=10^{8}$ with a real precision of 1000016 significant digits when $F(X)=v(x)$. We also checked that is still negative for $X=100000$ !, where $(.$.$) ! means the factorial function.$

\section{References}

1. Anderson, G., Vamanamurthy, M., Vuorinen, M.: Monotonicity Rules in Calculus. The American Mathematical Monthly 113(9), 805-816 (2006). DOI 10.1080/00029890.2006.11920367

2. Borwein, P.B., Choi, S., Rooney, B., Weirathmueller, A.: The Riemann Hypothesis: A Resource for the Afficionado and Virtuoso Alike, vol. 27. Springer Science \& Business Media (2008)

3. Choie, Y., Lichiardopol, N., Moree, P., Solé, P.: On Robin's criterion for the Riemann hypothesis. Journal de Théorie des Nombres de Bordeaux 19(2), 357-372 (2007). DOI 10.5802/jtnb.591

4. Ghosh, A.: An Asymptotic Formula for the Chebyshev Theta Function. arXiv preprint arXiv:1902.09231 (2019)

5. Kozma, L.: Useful Inequalities. http://www.lkozma.net/inequalities_cheat_sheet/ineq. pdf (2021). Accessed on 2022-01-11

6. Mertens, F.: Ein Beitrag zur analytischen Zahlentheorie. J. reine angew. Math. 1874(78), 46-62 (1874). DOI 10.1515/crll.1874.78.46. URL https://doi.org/10.1515/crll.1874.78.46

7. Nicolas, J.L.: Petites valeurs de la fonction d'Euler et hypothese de Riemann. Séminaire de Théorie des nombres DPP, Paris 82, 207-218 (1981)

8. Nicolas, J.L.: Petites valeurs de la fonction d'Euler. Journal of number theory 17(3), 375-388 (1983). DOI 10.1016/0022-314X(83)90055-0

9. Rosser, J.B., Schoenfeld, L.: Approximate Formulas for Some Functions of Prime Numbers. Illinois Journal of Mathematics 6(1), 64-94 (1962). DOI doi:10.1215/ijm/1255631807

10. Rosser, J.B., Schoenfeld, L.: Sharper Bounds for the Chebyshev Functions $\theta(\mathrm{x})$ and $\psi(\mathrm{x})$. Mathematics of computation pp. 243-269 (1975). DOI 10.1090/S0025-5718-1975-0457373-7

11. Von Koch, H.: Sur la distribution des nombres premiers. Acta Mathematica 24(1), 159 (1901) 